\title{
Drenaje del hematoma subdural crónico mediante agujeros de trépano.
}

Benito Naverac $H$.

Hospital Clínico Universitario Lozano Blesa de Zaragoza.

\section{Resumen}

En numerosas ocasiones el drenaje del hematoma subdural crónico se realiza mediante agujeros de trépano. Esta intervención es susceptible de ser realizada bajo anestesia local y sedación. En este artículo se responde a varias cuestiones que se plantean los anestesiólogos de guardia.

\section{Introducción}

En numerosas ocasiones el drenaje del hematoma subdural crónico se realiza mediante agujeros de trépano. Esta intervención es susceptible de ser realizada bajo anestesia local y sedación. En este artículo se responde a varias cuestiones que se plantean los anestesiólogos de guardia.

\section{¿Cuál es la etiopatogenia de este proceso?}

En una persona sana, el espacio subdural es prácticamente virtual, una de las razones es que el cerebro ocupa casi todo el volumen del cráneo. Como consecuencia del proceso de envejecimiento, se produce una atrofia cerebral, lo que conlleva una separación de la corteza cerebral del hueso, aumentando el espacio subdural, por donde pasan numerosas venas de drenaje. Si se produce un golpe fortuito se puede producir un sangrado, que si es pequeño puede no dar sintomatología.

Esta sangre se coagula y posteriormente con el paso de los días comienza a descomponerse. Como consecuencia del proceso de degradación, se forman substancias con poder osmótico, que atraen líquido (deshidratando el cerebro) lo cual aumenta y licúa el hematoma. Asimismo, se produce una reacción inflamatoria, con la aparición de una membrana densa de tejido vascularizado, la cual contribuye a cronificar el proceso. En el año 1857 Virchow, definió esta entidad como "paquimeningitis hemorrágica interna", lo que subraya la importancia de la reacción inflamatoria en la génesis de este proceso.

\section{¿Cuándo se puede hacer una anestesia local y sedación?}

Si el hematoma subdural crónico puede ser drenado a través de unos agujeros de trépano se puede optar por realizarlo bajo anestesia local y sedación. Si por el contrario requiere la realización de una craneotomía (hematoma multiloculado, presencia de múltiples membranas, calcificaciones, etc) será necesario la realización de una anestesia general.

$$
\begin{aligned}
& \text { ¿Cuáles son las ventajas de realizar el } \\
& \text { procedimiento bajo anestesia local y } \\
& \text { sedación? }
\end{aligned}
$$


La realización de este procedimiento bajo anestesia local representa todo un reto para el anestesiólogo. Los objetivos son conseguir un paciente consciente, colaborador, que mantenga una ventilación espontánea sin retención de carbónico y que no realice movimientos intempestivos durante el acto quirúrgico.

Todos estos objetivos son difíciles de conseguir, por lo que, en numerosas ocasiones, se prefiere optar por anestesia general. Sin embargo, la edad avanzada y la comorbilidad asociada hacen aconsejable realizar este procedimiento bajo anestesia local y sedación. Asimismo, nos permite realizar de forma inmediata una valoración neurológica, para evaluar la mejoría clínica del paciente.

\section{¿Qué agentes pueden usarse para la sedación?}

Se necesita un agente que proporcione sedación y analgesia. Es frecuente la combinación de varios agentes anestésicos, por lo que debemos vigilar que no se desarrolle una depresión respiratoria. Existen numerosas opciones de entre las cuales destacan:

- Administración de midazolam $(0,03-$ $0,05 \mathrm{mg} / \mathrm{Kg}$ ) e infusión continua de remifentanilo a $0,05-0,1 \quad \mu \mathrm{g} / \mathrm{kg} / \mathrm{min}$ (vigilancia estricta de la ventilación por posible aparición de depresión respiratoria y de rigidez de la pared torácica, de la frecuencia cardiaca y de la presión arterial.) Unos 5-7 min después del comienzo de la perfusión (tiempo necesario para preparar el campo quirúrgico) aparece miosis, momento en el que se le indica al cirujano que puede realizar la infiltración con anestesia local. Unos minutos después, cuando consideramos que el anestésico local ha conseguido hacer efecto se disminuye la infusión de remifentanilo a $0,025 \mu \mathrm{g} / \mathrm{kg} / \mathrm{min}$.
( http://revista.sedolor.es/pdf/2001_09 05.pdf).

- Se ha descrito la utilización de dexmedetomidina, un agonista $\alpha 2$ selectivo en este tipo de cirugías con buenos resultados, ya que tienen tiene la ventaja de tener efecto analgésico y de no producir depresión respiratoria (ver bibliografía adjunta, para consultar forma de administración). El problema con la dexmedetomidina es que, según ficha técnica, su uso sólo está aprobado para sedación en unidades de cuidados intensivos, lo cual no favorece su uso rutinario en el quirófano. Uno de los efectos secundarios frecuentes, cuando se administra en bolo es la bradicardia. Si se administra atropina para tratarla se corre el riesgo de que aparezca una crisis hipertensiva.

- Otras posibilidades es combinar midazolam con fentanilo (riesgo de depresión respiratoria.)

- Puede usarse también el propofol combinado con fentanilo o remifentanilo. Hay que dosificar adecuadamente los fármacos analgésicos, ya que puede aparecer agitación psicomotriz. Como ventajas destaca su efecto antiemético, y como inconvenientes el riesgo de depresión respiratoria y de movimientos intempestivos.

\section{¿Cómo podemos evitar la agitación?}

Las causas más comunes de agitación son el dolor y la ansiedad, y su control debe comenzar con reconocerlas adecuadamente para poder iniciar su tratamiento. El dolor se debe tratar con fármacos analgésicos y la ansiedad con ansiolíticos. Una inadecuada analgesia en un paciente somnoliento por los fármacos puede manifestarse con la aparición de agitación, lo que, si se trata equivocadamente con hipnóticos, puede aumentarse la desorientación, y el riesgo de depresión respiratoria. 
En general, la utilización de fármacos en perfusión continua que mantienen niveles estables, producen menos movimientos intraoperatorios y menos ajustes de dosis una vez alcanzado el nivel deseado de sedación.

¿Se debe utilizar la osmoterapia (Manitol o Suero Salino Hipertónico) en el hematoma subdural crónico?

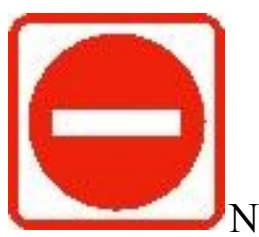

No deben utilizarse. Estas medidas tienen como objeto disminuir la PIC a base de deshidratar el parénquima cerebral. En esta situación el cerebro necesita reexpandirse, por lo que la administración de agentes osmóticos podría causar un efecto perjudicial. Al contrario, hay que hidratar al paciente de forma adecuada, para facilitar la recuperación del volumen cerebral.

Si no se recomienda la administración de corticoides en el TCE ¿Por qué se administra dexametasona a estos pacientes?

Como consecuencia del proceso inflamatorio se forma una membrana densa, de tejido vascularizado a lo largo del hematoma. Aunque su misión fundamental es reabsorber la sangre, provoca el efecto contrario, ya que segrega líquido, forma tabicaciones que dividen la cavidad del hematoma $y$ dificulta la reexpansión del cerebro una vez drenado.

La administración de dexametasona se basa en sus propiedades antiangiogénicas y antiinflamatorias sobre la membrana del coágulo subdural.

¿Se deben administrar profilaxis anticonvulsiva?
Es un tema controvertido. No hay evidencia científica clara para apoyar el uso rutinario de fármacos antiepilépticos en pacientes con hematoma subdural crónico.

\section{¿Es necesaria vigilancia postoperatoria en una Unidad de Cuidados Intensivos?}

Se debe de valorar cada caso de forma individual de forma cuidadosa. Si el paciente entra al quirófano con un Glasgow 15 y el procedimiento se realiza con anestesia local, podríamos enviarlo a la URPA, y de allí a la planta, sin pasar por la unidad de cuidados intensivos.

¿Se puede levantar el cabecero de la cama en estos pacientes? ¿Cómo se debe colocar del drenaje en el postoperatorio inmediato?

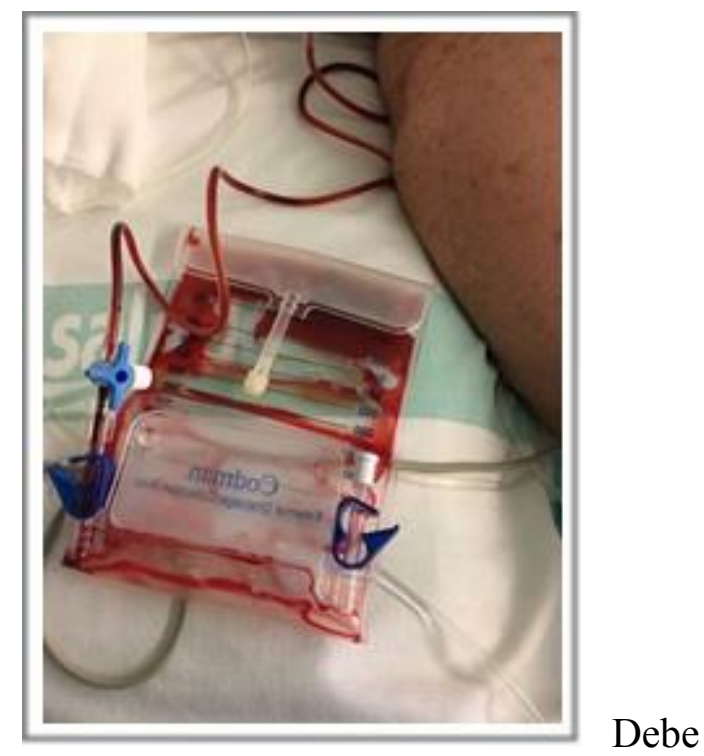

colocarse abierto situado algo por debajo del nivel del conducto auditivo externo de forma que se favorezca la salida de sangre, con el cabecero elevado a unos $30^{\mathrm{a}}$.

\section{Bibliografía}

- Yad R. Yadav, Vijay Parihar, Hemant Namdev, Jitin Bajaj. Chronic subdural hematoma. Asian Journal of 
Neurosurgery 330 Vol. 11, Issue 4, October-December 2016. ( $\underline{\text { PMC) }}$

- Bishnoi V, Kumar B, Bhagat H, Salunke P, Bishnoi S. Comparison of Dexmedetomidine Versus MidazolamFentanyl Combination for Monitored Anesthesia Care During Burr-Hole Surgery for Chronic Subdural Hematoma. J Neurosurg Anesthesiol. Volume 28, Number 2, April 2016. (PubMed)

- Wenming Wang, Lei Feng, Fenfen Bai, Zongwang Zhang, Yong Zhao,2, Chunguang Ren. The Safety and Efficacy of Dexmedetomidine vs. Sufentanil in Monitored Anesthesia Care during Burr-Hole Surgery for Chronic Subdural Hematoma: A Retrospective Clinical Trial. Front Pharmacol 2016.Nov 3;7:410. eCollection 2016. (HTML) (PDF)
- http://revista.sedolor.es/pdf/2001_09_0 5.pdf

- Ratilal BO, Pappamikail L, Costa J, Sampaio C. Anticonvulsants for preventing seizures in patients with chronic subdural haematoma. Cochrane Database of Systematic Reviews 2013, Issue 6. Art. No.: CD004893. DOI: 10.1002/14651858.CD004893.pub3

Correspondencia al autor

Helena Benito Naverac helenabenito@yahoo.es FEA de Anestesiología y Reanimación. Hospital Clínico Universitario Lozano Blesa de Zaragoza.

Aceptado para blog en mayo de 2018. 